\title{
Empirical Formalism for the Phantom Scatter Factor of Small Fields: Using Different Density Media
}

\author{
Saed J. Al Atawneh ${ }^{1,2 *}$ \\ ${ }^{1}$ Department of Radiation Oncology, King Hussein Cancer Center, Amman, Jordan \\ ${ }^{2}$ Department of Physics, Faculity of Science, Al-Balqa Applied University, Al-Salt, Jordan \\ Email: *saed.al-atawneh|@atomki.hu
}

How to cite this paper: Al Atawneh, S.J. (2022) Empirical Formalism for the Phantom Scatter Factor of Small Fields: Using Different Density Media. International Journal of Medical Physics, Clinical Engineering and Radiation Oncology, 11, 36-47.

https://doi.org/10.4236/ijmpcero.2022.111004

Received: December 13, 2021

Accepted: January 24, 2022

Published: January 27, 2022

Copyright $\odot 2022$ by author(s) and Scientific Research Publishing Inc. This work is licensed under the Creative Commons Attribution International License (CC BY 4.0).

http://creativecommons.org/licenses/by/4.0/

\begin{abstract}
We present the Empirical Formula (EF) to calculate the phantom scatter factor, $S_{p}$, of small radiation fields under charge particle dis-equilibrium conditions. The Empirical Formula (EF) was verified by examining the calculated data with experimentally measured data utilizing the anthropomorphic phantom in twelve different combinations of beam entry and point location, where the value for $S_{p}$ per tissue composition was within $3 \%$ in $8 / 12$ cases, $5 \%$ in $1 / 12$ cases, and $10 \%$ in $3 / 12$ cases. Our results showed a good agreement with experimental data to less than $1 \%$ when the ion chamber was surrounded by the homogeneous tissue, whether lung, soft tissue, or bone. Indicating that the prediction of the equation is valid, and it can be reliably used for phantom scatter factor calculation for different homogeneous media under charge particle disequilibrium conditions.
\end{abstract}

\section{Keywords}

Small Field Dosimetry, Anthropomorphic Study, Phantom Scatter Factor, Dosimetry

\section{Introduction}

Radiation therapy techniques have been accelerated to develop new treatment methods that might be considered more precise and more secure than ancient ones. Intensity Modulated Radiation Therapy (IMRT) and Volumetric Modulated Arc Therapy (VMAT) are introduced to provide accurate treatment for cancer patients and to minimize the dose on the surrounding normal tissue [1]-[6], consequently, the concept of "the small field dosimetry" came to use [3] [7] [8] [9]. 
Generally, these advanced techniques are utilized in a small field or segment deliveries which are dis-equilibrium conditions [10]. A brief definition of a small field is still unclear. Normally, it is considered from the field dimension which is less than the lateral range of a charged particle [9] [11] [12] [13]. Dosimetry of small beams suffers from the lack of lateral Charged Particle Equilibrium (CPE) [7]. As such, they are easily affected by: 1) the size of the collimator aperture(r); 2) the size of the measuring device; 3) the energy of photons; and 4) the characteristic of tissues (homogeneous or heterogeneous tissue). The secondary electrons produced from megavoltage photons, as they interact with the tissue, have a considerable range measured in centimeters in a water density environment. This range gets prolonged when electrons travel through a low-density medium, such as the lung. Compared to the field size, the lateral range of the electrons, rather than the forward range, is the critical parameter to the Charged Particle Equilibrium (CPE).

In the small field, the total scatters factor $\left(S_{c, p}\right)$ is underestimated. Therefore, the radiation dose is overestimated "i.e., overdose". Many research had been associated with small field dosimetry [1] [2] [3] [7] [8] [12] [14] [15] [16] [17], using different detectors under charge particle dis-equilibrium conditions to provide the same conclusion; the smaller the detector the better (more accurate) the total scatter factor readings are, none of these studies were concerned about the effect of densities variation on the total scatter factor, especially the phantom scatter factor $\left(S_{p}\right)$ parameter.

This work is geared towards utilizing different-sized detectors in various media such as air, wood, water, and acrylic to locate the impact of the change in medium densities on the phantom scatter factor parameter, particularly under charge particle dis-equilibrium condition (Dis-CPE) [11] [12] [15]. Along this line, in this paper, we present a mathematical expression (Empirical Formal) to calculate the phantom scatter factor $\left(\mathrm{S}_{\mathrm{p}}\right)$ of small radiation fields under extreme conditions such as the charge particle dis-equilibrium.

\section{Materials and Methodology}

\subsection{Materials}

Four different media were used in this study, air measurements were performed using mini-Phantom for collimator scatter factor " $S_{c}$ ", Water Phantom $\left(1 \mathrm{~g} / \mathrm{cm}^{3}\right)$, Wood Phantom $\left(0.63 \mathrm{~g} / \mathrm{cm}^{3}\right)$, and Acrylic phantom $\left(1.136 \mathrm{~g} / \mathrm{cm}^{3}\right)$. All measurements were accomplished by three extraordinary ion chambers, see Table 1.

The linear accelerator (Elekta Synergy, model number (151150)) was utilized, it produces three photon beam energies; 6, 10, and $15 \mathrm{MV}$. Linear accelerator was attached by Apex Collimator, is an add-on MLC device that is capable of delivering very small conformal fields such as $0.98 \times 0.98 \mathrm{~cm}^{2}$. For the validation process, CT-Simulator, Brilliance Big Bore-Phillips model, 64-Slice with $85 \mathrm{~cm}$ aperture bore, was used. The anthropomorphic phantom was handled as a patient. It was 
Table 1. Characteristics of detectors used in this study.

\begin{tabular}{ccccc}
\hline Detectors & Detector Type & Sensitive Volume $(\mathrm{mm})$ & Volume & Material \\
\hline PTW 30010 Wellhofer Farmer & Gas Filled & Radius 3.05 $(\mathrm{mm})$, Length 23 $(\mathrm{mm})$ & $0.65\left(\mathrm{~cm}^{3}\right)$ & Acrylic Wall, Graphited \\
PTW 31010 Semiflex & Gas Filled & Radius 2.75 $(\mathrm{mm})$, Length $6.5(\mathrm{~mm})$ & $0.125\left(\mathrm{~cm}^{3}\right)$ & Acrylic Wall, Graphited \\
PTW 31023 Pinpoint & Gas Filled & Radius 1.0 $(\mathrm{mm})$, Length 5.0 $(\mathrm{mm})$ & $0.015\left(\mathrm{~cm}^{3}\right)$ & Acrylic Wall, Graphited \\
\hline
\end{tabular}

the first CT scan, and then its image was transferred to the treatment planning system (Monaco) where the radiation treatment plan was done before it was measured on the linac.

\subsection{Methodology}

\subsubsection{The Total Scatter Factor, $S_{c, p}$, and Collimator Scatter, $S_{c}$, Measurements}

The phantom was placed such that the distance from the source to the surface (SSD) was $100 \mathrm{~cm}$, where the total scatter factor $\left(S_{c, p}\right)$, a ratio of the absorbed dose at the reference depth for given field size, to the dose at the same depth for the reference field size, was measured at the reference depth, which for this work was $10 \mathrm{~cm}$ in depth. The total scatter factor is given by Equation (1):

$$
S_{c, p}=\Omega_{f_{\text {clin }} Q_{\text {msr }}}^{f_{\text {clis }}, f_{\text {ms }}}=\frac{D_{W, Q_{\text {clin }}}^{f_{\text {clin }}}}{D_{W, Q_{\text {msr }}}^{f_{\text {msr }}}}
$$

where: $D_{W, Q_{\text {clin }}}^{f_{\text {clin }}}$ and $D_{W, Q_{\text {msr }}}^{f_{\text {msr }}}$ are the absorbed dose to water in the clinical field $f_{\text {clin }}$ with beam quality $Q_{\text {clin }}$ and absorbed dose to water in the machine specific reference field $f_{\text {msr }}$ with beam quality $Q_{\text {msr }}$, respectively [7] [8]. For collimator scatter factor $\left(S_{c}\right)$, the ratio of the output in the air for a given field to that for a reference field, measurements, the buildup caps were used "a small phantom" and the ion chamber was positioned such that the distance from the source to the detector (SDD) is $110 \mathrm{~cm}$.

During all measurements, the temperature and pressure were taken to be (902 $\mathrm{hPa}$ ) and (20 Celsius) on average. 100 Monitor Unit (MU) was delivered for all data measurements. The chamber is set up within the phantoms such that its axis should always be perpendicular to the central beam axis "CAX", and the center of the ionization chamber assumed to be located at the measurement depth of 10 $\mathrm{cm}$, this depth was kept constant while changing the field size for each measurement [1] [7] [8] [14]. Readings for the $S_{c p}$ was taken for MLC-shaped fields starting from $\left(9.8 \times 9.8 \mathrm{~cm}^{2}\right)$ down to $\left(0.98 \times 0.98 \mathrm{~cm}^{2}\right)$ and then normalized to the reference field size of $\left(9.8 \times 9.8 \mathrm{~cm}^{2}\right)$.

\subsubsection{Anthropomorphic Phantom Measurements}

The anthropomorphic phantom was used to affirm this work under complex anatomical geometry. $S_{c, p}$ was measured for clinical field sizes $\left(5.39 \times 5.39 \mathrm{~cm}^{2}\right)$ and reference field size $\left(9.8 \times 9.8 \mathrm{~cm}^{2}\right)$ by the Wellhofer Farmer ionization chamber, and through the lung, soft tissue, and bone "vertebra" sites utilizing one pho- 
ton energy (6 MV). The phantom was set up and aligned with the lasers on the table of the CT-Simulator, scanned, and the images were sent to the Monaco treatment planning system (TPS), treatment plans were made for two sites in the lung, central and periphery sites, for the vertebral bone and the mediastinal region also. Each region was planned with three beams; Anterior-Posterior (AP), Posterior-Anterior (PA), and Left-Lateral (LT), and each beam delivered $100 \mathrm{cGy}$.

\section{Result and Discussion}

\subsection{Effect of Ion Chamber Size and Medium Density Variation on the Total Scatter Factor}

In this part, the ion chambers sizes and media densities effect were considered extensively using three different ion chambers.

Figure 1 shows the $S_{c, p}$ 's at the large fields $\left(>4.41 \mathrm{~cm}^{2}\right)$ for different media and ion chambers are similar with less than $1 \%$ due to the presence of $(\mathrm{CPE})$ at the point of measurement [18]. At the small field size $\left(<4.41 \mathrm{~cm}^{2}\right)$, the $S_{c, p}$ readings in the water for Farmer-Semiflex and Farmer-Pinpoint ion chambers were deviated

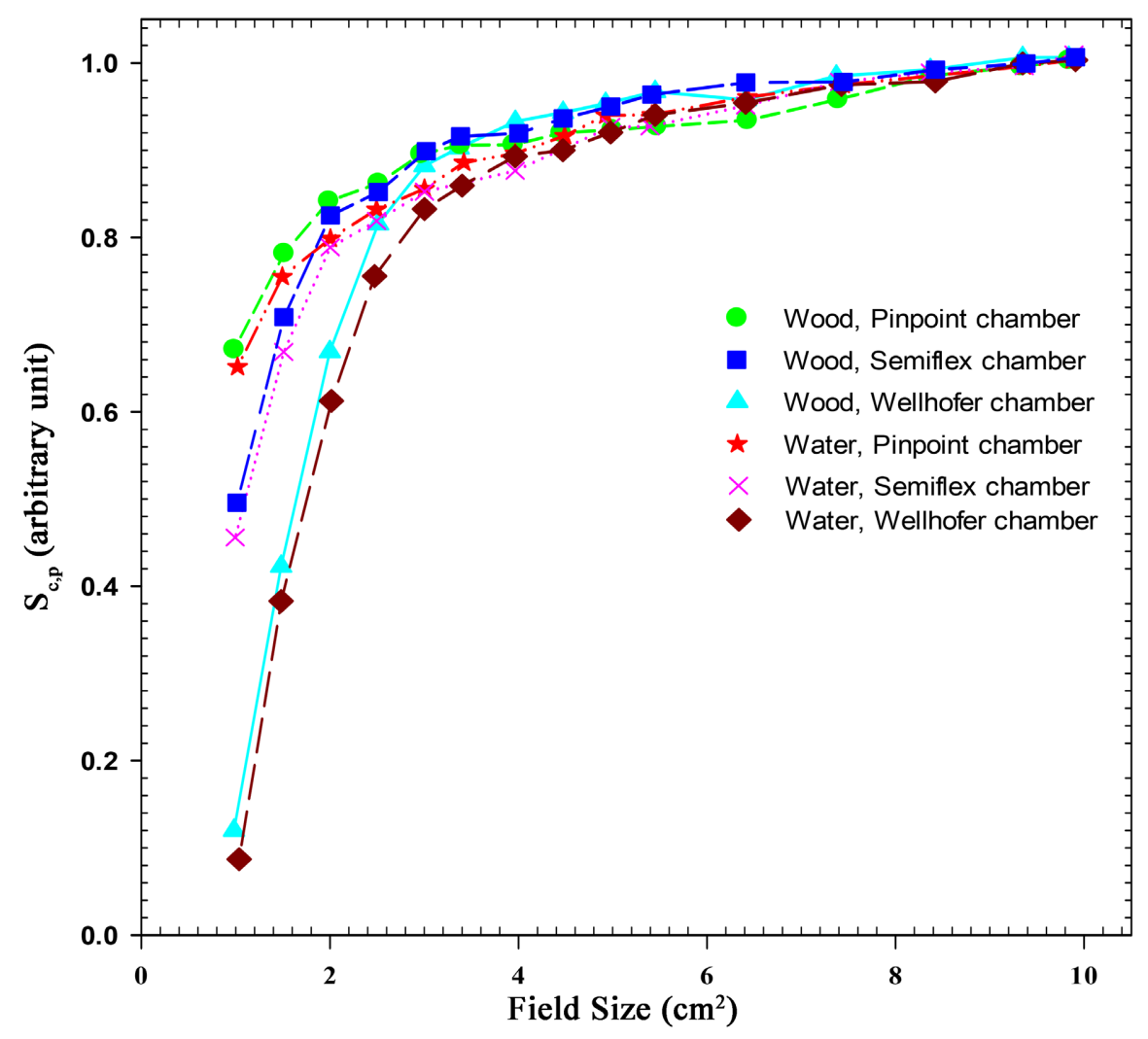

Figure 1. The total scatter factor $\left(S_{c, p}\right)$ as a function of field size for different ion chambers and media. Green circle-dash line: the $S_{c, p}$ in the wood media measured by Pinpoint ion chamber; Blue squre-dash line: the $S_{c, p}$ in the wood media measured by semiflex ion chamber; Cyan triangle-solid line: the $S_{c, p}$ in wood media measured by wellhofer ion chamber; Red stars-dash line: the $S_{c, p}$ in water media measured by Pinpoint ion chamber; Pink cross-dots: the $S_{c, p}$ in water media measured by semiflex ion chamber; Dk Red diamond-dash line: the $S_{c, p}$ in water media utilizing wellhofer ion chamber. 
by $37 \%$ and $43 \%$ respectively, due to loss of CPE at the point of interest [2] [14].

$S_{c, p}$ for the small field that measured by large chamber will be underestimated "i.e., less resolution", this in turn, will lead to an overestimation in the Monitor Unit calculation (MU), and thus over-dosing the patient [9]. Figure 1 shows the total scatter factor readings as a function of field size.

\subsection{Estimating Formula for $S_{p}$}

For the Estimating Formula, the total scatter factor that was measured, where the Pinpoint ion chamber shall only consider. So; the phantom scatter factor $\left(S_{p}\right)$ was calculated based on the total scatter factor measurements using Pinpoint ion chamber through wood, water, and acrylic phantom according to Equation (2):

$$
S_{p}(r) * \varepsilon\left(s ; z ; d_{\text {ref }}\right)=\frac{S(r)_{c, p}}{S(r)_{c}}
$$

where: $\varepsilon\left(s ; z ; d_{\text {ref }}\right)=\beta(s ; z ; d) / \beta\left(s ; z ; d_{\text {ref }}\right)$ is the electron dis-equilibrium factor ( $\varepsilon=1$ for adequate depths and positions adequately far from the edges of the field). We assumed that $d_{\text {ref }}$ is sufficiently large to establish electron equilibrium and shield from contamination electrons [7] [8]. Figure 2 shows the $S_{p}$ values of the three media (wood, water, and acrylic) as a function of field size.

Each curve was fitted by means of an exponential relation due to the attenuation nature of photons in the media [19], and represented by Equation (3):

$$
S(r)_{p, \text { open }}=y_{0}+A_{1}\left(1-\mathrm{e}^{-k_{1} * r}\right)+A_{2}\left(1-\mathrm{e}^{-k_{2} * r}\right)
$$

where: $A_{1}, A_{2}, k_{1}, k_{2}$, and $y_{0}$ are fitting parameters that depend on the density

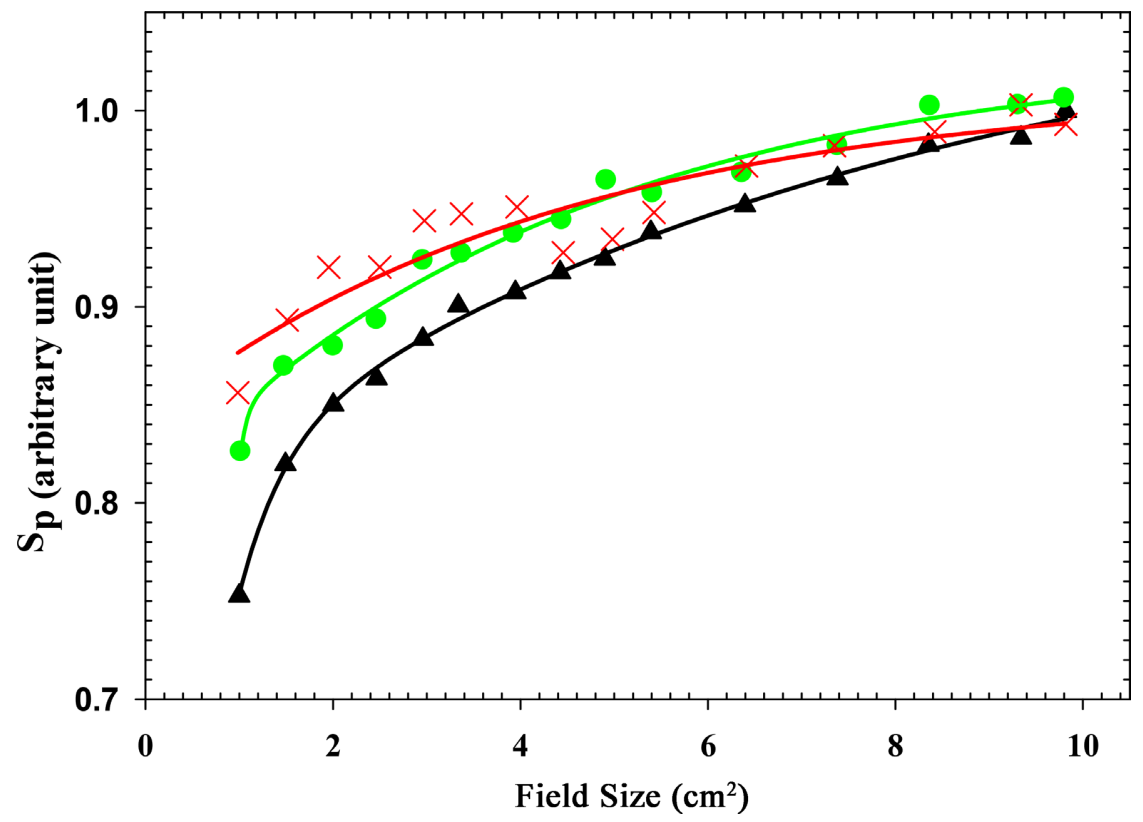

Figure 2. The phantom scatter factor $\left(S_{p}\right)$ as a function of field size for pinpoint ion chamber. Red cross: the phantom scatter factor in the wood media; Green circle: the phantom scatter factor in the water media; Black triangle: the phantom scatter factor in the acrylic media. 
(medium).

The first term of Equation (3), $y_{0}$, represents the phantom scatter factor at the zero field size, and the second term represents an exponential fitting to small field size $<4.41 \mathrm{~cm}^{2}$, while the last term represents an exponential fitting to the large field size $>4.41 \mathrm{~cm}^{2}$.

The water medium was used for the total output factor measurement as a base value to all different media with $\pm 2 \%$ deviation for the field sizes $\geq 2.94 \times 2.94$ $\mathrm{cm}^{2}$, and with $3 \%$ deviation for the field sizes $1.47<r<2.94 \mathrm{~cm}^{2}$, whilst the ultra small field sizes $<1.47 \mathrm{~cm}^{2}$ were deviated by $\pm 5 \%$ from the baseline value emphasis that the medium density impact appears clearly at the small radiation beams and should be taken into our account.

\subsection{The Output Factor Ratio}

Now, the total scatters factor ratio or let say, the total output factor ratio (OFR) is introduced, which is a ratio of total scatters factor $S_{c, p}$ in any medium relative to that in water media, see Equation (4):

$$
\mathrm{OFR}_{\text {water }}^{\text {medium }}=\frac{\left(S_{c, p}\right)_{\text {medium }}}{\left(S_{c, p}\right)_{\text {water }}} \approx \frac{\left(S_{c}(r) \times S_{p}(r)\right)_{\text {medium }}}{\left(S_{c}(r) \times S_{p}(r)\right)_{\text {water }}}
$$

As noticed, the ratio of the phantom scatter factor $S_{p}(r)_{\text {medium }} / S_{p}(r)_{\text {water }}$, is directly proportional to the ratio of charge collected providing that the same chamber and the same irradiation geometry between water and medium. OFR was plotted as a function of field size for two different media and two special ion chambers (see Figure 3).

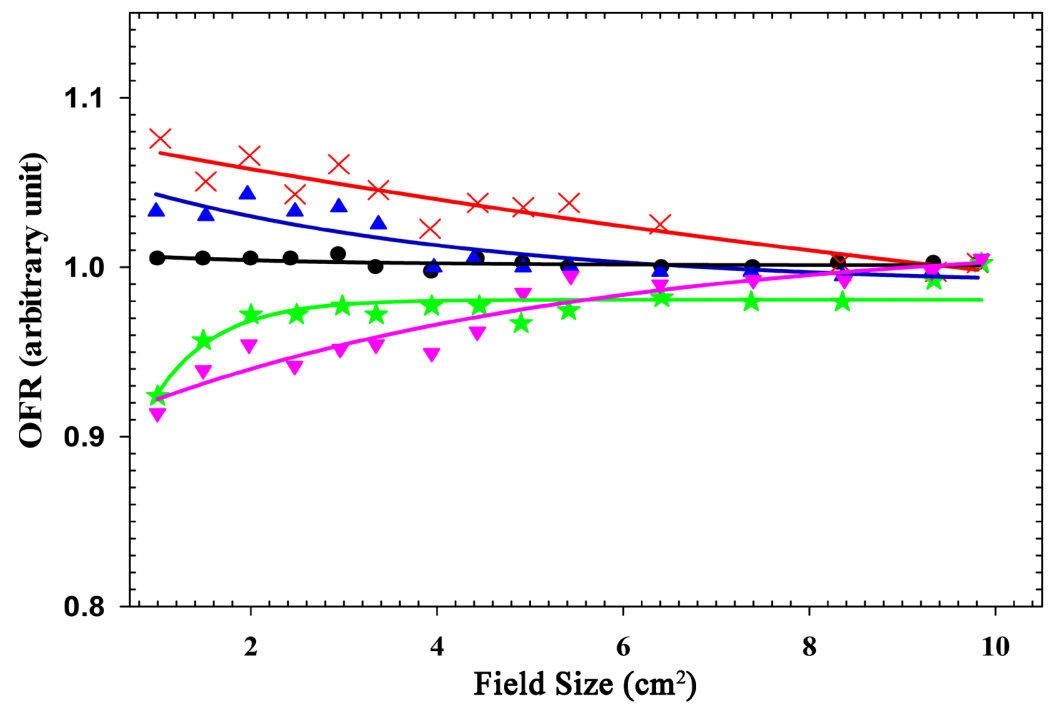

Figure 3. The total Output Factor Ratio (OFR) as a function of filed size for two different media and two different ion chambers. Red cross-dash line: the OFR in the wood media measured by semiflex ion chamber; Blue triangle-up-dash line: the OFR in wood media measured by Pinpoint ion chamber; Black circle-solid line: the OFR in water media; Pink triangle-down-dots: the OFR in acrylic media measured by semiflex ion chamber; Green stars-dash line: the OFR in acrylic media measured by Pinpoint ion chamber. 
OFR curves from Figure 3 are seen to be straight with value of 1 for broad beams and to slightly curve upward for low-density medium or downward for high-density medium to $\pm 5 \%$ variation; consequently, the exponential function was utilized to fit this behavior and relate the OFR to the field size, $r$, and to the density in the form of $\mathrm{OFR}_{w}^{m}=Q \pm P \mathrm{e}^{-K r}$, where $Q, P$, and $K$ are fitting parameters, where $P$ is negative for high-density medium.

\subsection{The Ion Chamber Volume Effect}

The presence of a foreign object like the ion chamber through the homogenous medium will perturb the electron flux, especially near the ion chamber location [1] [2] [3] [11] [14]. Figure 4 showed that the estimated quantity of the perturbation produces by ion chambers cavity through a homogeneous medium. The chamber volume versus the OFR was plotted for different media and the extrapolation to zero ion chamber size was carried out, see in Figure 4(a) and Figure 4(b).

Extrapolating each curve in Figure 4(a) and Figure 4(b) until it intersects with the Y-axis, yields the OFR ratio with zero chamber size, i.e., independent of ion chamber size, in the form of $\operatorname{OFR}_{W}^{M}=(A+B * \text { ion chamber volume })_{\text {field size }}$,

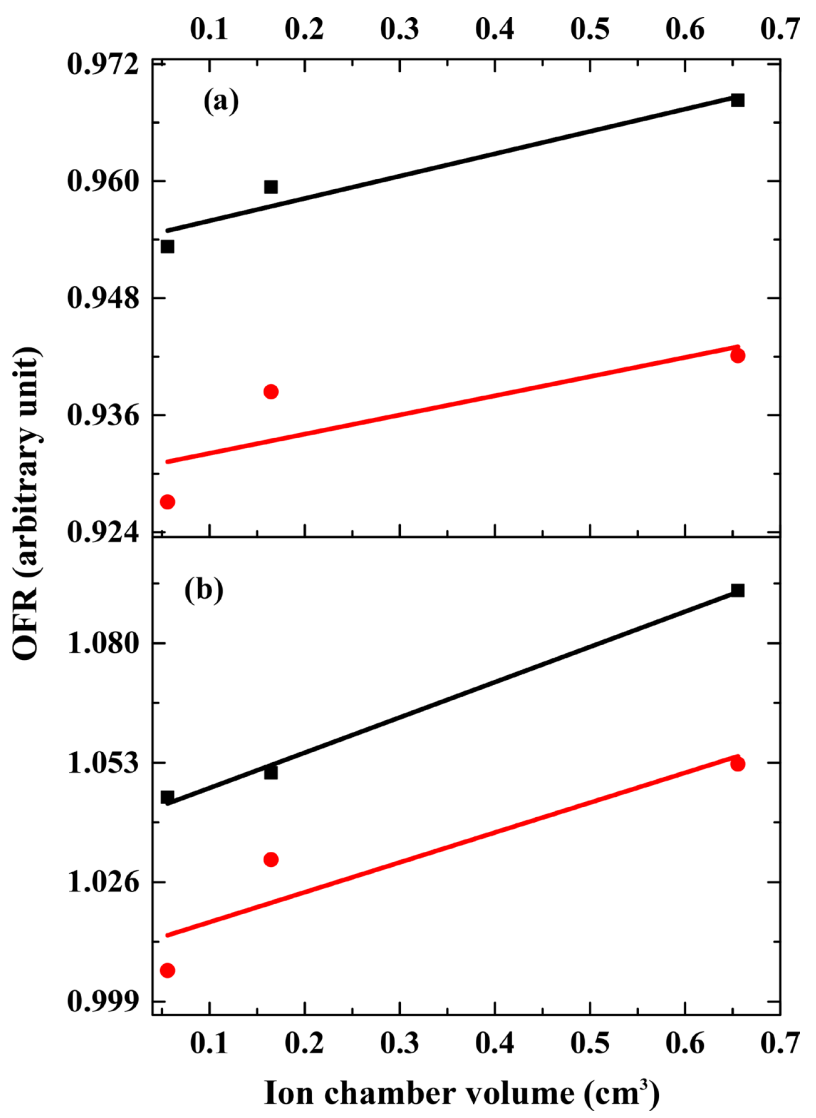

Figure 4. The Output Factor Ratio (OFR) as a function of the ion chamber volumes for different filed sizes. a) Black Square: the OFR measured in wood medium for $0.98 \mathrm{~cm}^{2}$ filed size; Red circle: the OFR measured in wood medium for $1.47 \mathrm{~cm}^{2}$ filed size; b) Black square: the OFR measured in Acrylic media for $1.47 \mathrm{~cm}^{2}$ filed size; Red circle: the OFR measured in acrylic media for $0.98 \mathrm{~cm}^{2}$ filed size. 
where the $A$ and $B$ are fitting parameters; therefore, the OFR values could be found for small fields without chamber perturbations impact.

\subsection{Empirical Formula (EF) for $S_{p}$}

Starting from Equation (5), the relative output factor could be used to estimate the phantom scatter value as follow:

$$
S(r)_{p, \text { medium }}=S(r)_{p, \text { water }} \cdot(\text { O.F.R })_{w}^{m}
$$

By substituting in the analytical equations for the water scatter phantom $S(r)_{p \text {,water }}$ and the output factor ratio, OFR, in Equation (5), we get:

$$
S(r)_{p, m}=\left(y_{0}+A_{1}\left(1-\mathrm{e}^{-k_{1} * r}\right)+A_{2}\left(1-\mathrm{e}^{-k_{2} * r}\right)\right)_{p, w} \cdot\left(Q \pm P \mathrm{e}^{-K * r}\right)_{w}^{m}
$$

where $y_{0}, A_{1}, A_{2}, k_{1}, k_{2}, Q$ and $P$ are values depending on medium density.

Or:

$$
S(r)_{p, m}=\left(y_{0}+A_{1}\left(1-\mathrm{e}^{-k_{1} * r}\right)+A_{2}\left(1-\mathrm{e}^{-k_{2} * r}\right)\right)_{p, w} \cdot(A \pm B \cdot \text { ion chamber vol })_{r}
$$

\section{Experiment Validation}

Empirical Formula (EF) for the phantom scatter factor was tested for various media, mediastinum, and two positions in the lung (middle and periphery) as well as tissue surrounded by bone (spinal cord). For each location, three beam geometries were used, AP, PA, and Lt Lat beam. The idea of which is to change the composition of the tissue that the beam goes through before it reaches the point of interest.

\subsection{Lung Site}

Starting with the low-density material, lung density is equal to $0.21 \mathrm{~g} / \mathrm{cm}^{3}$, the lung dose was planned and measured at two sites; at the center and peripherial sites of the lung, each site had three beams irradiating as mentioned previously.

Table 2 shows the calculated phantom scatter factor $\left(S_{p, \text { calculated }}\right)$ by EF compared to measured phantom scatter factor $\left(S_{p, \text { measured }}\right)$ by Pinpoint ion chambers, the central lung site.

As shown, the EF yield just a single estimation of $S_{p}$ and it matches very well with the value obtained for the Lt Lat and PA beams, and furthermore it ought to be noticed that the fitting parameters were obtained from wood phantom $(\rho=$ $\left.0.63 \mathrm{~g} / \mathrm{cm}^{3}\right)$ that utilize to represent the lung medium $\left(\rho=0.21 \mathrm{~g} / \mathrm{cm}^{3}\right)$, however,

Table 2. The phantom scatter factors $S_{p}$ for central lung, anthropomorphic phantom.

\begin{tabular}{cccc}
\hline Beams & $S_{p \text { Measured }}$ & $S_{p \text { Calc }}$ & \%error \\
\hline AP & 0.91 & 1.00 & -9.46 \\
Lt Lat & 1.00 & 1.00 & 0.28 \\
PA & 0.98 & 1.00 & -2.32 \\
\hline
\end{tabular}


it still applies well in this particular case. For the periphery lung site Table 3 was introduced.

As noticed, the ion chamber was located exceptionally close to the soft tissue. As such, the phantom scatter factor that chamber measures "sees" is greatly influenced by the presence of the soft tissue. This scenario is not taken into account with $\mathrm{EF}$, which predicts the phantom scatter factor when the chamber is encompassed by enough homogeneous medium.

\subsection{Mediastinum Site}

The mediastinum site was represented with a normal density equal to $1.01 \mathrm{~g} / \mathrm{cm}^{3}$, further measurements were accomplished via the anthropomorphic phantom and pinpoint ion chamber. The $S_{p, \text { calc }}$ was carried out using equation 7 and compared with measured one, see Table 4.

Table 4 shows that the expected values of $S_{p \text {,calc }}$ was within $1 \%$ from measurement values because of the ion chamber was completely surrounded by the soft tissue which validates equation 7 for $S_{p}$ in the soft-tissue medium.

\subsection{Vertebral Bone Site}

Last but not the least, the $S_{p}$ for the high-density material, vertebral bone with density equal to $1.59 \mathrm{~g} / \mathrm{cm}^{3}$, was measured under the same previous condition and the $S_{p \text {,calc }}$ was calculated by Equation (7). Both values were compared as shown in Table 5.

Table 3. Phantom scatter factor for periphery lung, anthropomorphic phantom.

\begin{tabular}{cccc}
\hline Beams & $\mathcal{S}_{p \text { Measured }}$ & $\mathcal{S}_{p \text { Calc }}$ & \%error \\
\hline AP & 0.93 & 1.00 & -7.50 \\
Lt Lat & 0.97 & 1.00 & -3.3 \\
PA & 0.90 & 1.00 & -9.62 \\
\hline
\end{tabular}

Table 4. Phantom scatter factor for mediastinum, anthropomorphic phantom.

\begin{tabular}{cccc}
\hline Beams & $\mathcal{S}_{P, \text { Measured }}$ & $\mathcal{S}_{P, \text { Calc }}$ & \%error \\
\hline AP & 0.97 & 0.96 & 0.96 \\
Lt Lat & 0.97 & 0.96 & 1.09 \\
PA & 0.96 & 0.96 & 0.68 \\
\hline
\end{tabular}

Table 5. Phantom scatter factor for vertebral bone, anthropomorphic phantom.

\begin{tabular}{cccc}
\hline Beams & $\mathcal{S}_{p \text { Measured }}$ & $\mathcal{S}_{p \text { Calc }}$ & \%error \\
\hline AP & 0.94 & 0.93 & 1.23 \\
Lt Lat & 0.94 & 0.93 & 1.27 \\
PA & 0.98 & 0.93 & 4.92 \\
\hline
\end{tabular}


The measuring values are relatively close to the calculated one with a maximum deviation of $4.92 \%$ for the worst case scenario. The EF was based on the fitting parameters that extracting from acrylic media, density is equal to 1.136 $\mathrm{g} / \mathrm{cm}^{3}$, to represent the bone media, which might be a main source of this deviation. Furthermore, the AP beam showed a small deviation of $0.04 \%$ from the PA beam. The phantom scatters factor that the chamber measured from the AP field "sees" is highly affected by the presence of lung tissue in front of the vertebra tissue.

The measurements with the anthropomorphic phantom have validated the EF to calculate $S_{p}$. The values agreed within less than $1 \%$ when the chamber was surrounded by a homogeneous medium (all lung, all tissue, or all bone). When the beam arrangements and/or the location of the chamber resulted in the beam passing through variable tissues the results were not as good. This is expected because the EF has been extracted from homogeneous composition media.

\section{Conclusion}

We presented the Empirical Formula (EF) to calculate the total scatter factor under extreme conditions. The equations' results were confirmed using measurements from homogeneous media as well as the anthropomorphic phantom in 12 different combinations of beam entrances and point positions. The phantom scatter factors were within $3 \%$ in $8 / 12$ cases, $5 \%$ in $1 / 12$ cases, and $10 \%$ in $3 / 12$ cases. We found that the EF can be precisely employed for the $S_{p}$ calculation for various homogeneous media. However, further research is needed to provide a complete picture via an inhomogeneous medium under charge particle dis-equilibrium conditions. Our approach, on the other hand, can be utilized for $S_{p}$-factor modeling or even estimating the $S_{p}$-value for small radiation beams in diverse media without the need for additional measurements.

\section{Conflicts of Interest}

The author declares no conflicts of interest regarding the publication of this paper.

\section{References}

[1] Ding, G.X., Duggan, D.M. and Coffey, C.W. (2006) Commissioning Stereotactic Radiosurgery Beams Using Both Experimental and Theoretical Methods. Physics in Medicine \& Biology, 51, 2549-2566. https://doi.org/10.1088/0031-9155/51/10/013

[2] Sauer, O.A. and Wilbert, J. (2007) Measurement of Output Factors for Small Photon Beams. Medical Physics, 34, 1983-1988. https://doi.org/10.1118/1.2734383

[3] Klein, D.M., et al. (2010) Measuring Output Factors of Small Fields Formed by Collimator Jaws and Multileaf Collimator Using Plastic Scintillation Detectors. Medical Physics, 37, 5541-5549. https://doi.org/10.1118/1.3488981

[4] Benmakhlouf, H., Sempau, J. and Andreo, P. (2014) Output Correction Factors for Nine Small Field Detectors in 6 MV Radiation Therapy Photon Beams: A PENELOPE Monte Carlo Study. Medical Physics, 41, Article ID: 041711. 
https://doi.org/10.1118/1.4868695

[5] Francescon, P., Cora, S. and Cavedon, C. (2008) Total Scatter Factors of Small Beams: A Multidetector and Monte Carlo Study. Medical Physics, 35, 504-513. https://doi.org/10.1118/1.2828195

[6] Back, M., et al. (2013) Dosimetric Improvements Utilising Intensity Modulated Radiation Therapy for Patients with Glioblastoma Multiforme. Journal of Cancer Therapy, 4, 18-24. https://doi.org/10.4236/jct.2013.411A003

[7] Huq, M.S., et al. (2018) A Dosimetric Evaluation of the IAEA-AAPM TRS483 Code of Practice for Dosimetry of Small Static Fields Used in Conventional Linac Beams and Comparison with IAEA TRS-398, AAPM TG51, and TG51 Addendum Protocols. Medical Physics, 45, 4257-4273. https://doi.org/10.1002/mp.13092

[8] Zhu, T.C., et al. (2009) Report of AAPM Therapy Physics Committee Task Group 74: In-Air Output Ratio, for Megavoltage Photon Beams. Medical Physics, 36, $5261-$ 5291. https://doi.org/10.1118/1.3227367

[9] Masanga, W., et al. (2016) Determination of Small field Output Factors in 6 and 10 MV Flattening Filter Free Photon Beams Using Various Detectors. Journal of Physics: Conference Series, 694, Article ID: 012027. https://doi.org/10.1088/1742-6596/694/1/012027

[10] Ozdemir, H., Tuncel, N. and Kizildağ, A. (2017) The Impact of Small Field's Off-Set on Output Factor in Intensity Modulated Radiation Treatment. International Journal of Medical Physics, Clinical Engineering and Radiation Oncology, 6, 433-444. https://doi.org/10.4236/ijmpcero.2017.64039

[11] Haider, T.K. and El-Khatib, E.E. (1994) Measurements of Phantom Scatter Factors for Small Field Sizes in High Energy X Rays. Medical Physics, 21, 663-666.

https://doi.org/10.1118/1.597319

[12] Charles, P.H., et al. (2014) A Practical and Theoretical Definition of very Small Field Size for Radiotherapy Output Factor Measurements. Medical Physics, 41, Article ID: 041707. https://doi.org/10.1118/1.4868461

[13] Klingman, E. (2021) A Self-Linking Field Formalism. Journal of Modern Physics, 12, 440-452. https://doi.org/10.4236/jmp.2021.124031

[14] Das, I.J., Ding, G.X. and Ahnesjo, A. (2008) Small Fields: Nonequilibrium Radiation Dosimetry. Medical Physics, 35, 206-215. https://doi.org/10.1118/1.2815356

[15] Bassinet, C., et al. (2013) Small Fields Output Factors Measurements and Correction Factors Determination for Several Detectors for a CyberKnife(R) and Linear Accelerators Equipped with MicroMLC and Circular Cones. Medical Physics, 40, Article ID: 071725. https://doi.org/10.1118/1.4811139

[16] Nasir, M., et al. (2017) Measurement and Analysis of PDDs Profile and Output Factors for Small Field Sizes by cc13 and Micro-Chamber cc01. International Journal of Medical Physics, Clinical Engineering and Radiation Oncology, 6, 36-56. https://doi.org/10.4236/ijmpcero.2017.61005

[17] Islam, M.R., Watabe, H. and Andreoli, S. (2020) Measurement and Comparison of Output Factors Using Two Detectors for NOVAC7 IntraOperative Radiotherapy Accelerator. International Journal of Medical Physics, Clinical Engineering and Radiation Oncology, 9, 52-61. https://doi.org/10.4236/ijmpcero.2020.92006

[18] Hrbacek, J., Lang, S. and Klock, S. (2011) Commissioning of Photon Beams of a Flattening Filter-Free Linear Accelerator and the Accuracy of Beam Modeling Using an Anisotropic Analytical Algorithm. International Journal of Radiation Oncology, Biology, Physics, 80, 1228-1237. https://doi.org/10.1016/j.ijrobp.2010.09.050 
[19] Laub, W.U. and Wong, T. (2003) The Volume Effect of Detectors in the Dosimetry of Small Fields Used in IMRT. Medical Physics, 30, 341-347.

https://doi.org/10.1118/1.1544678 